\title{
Comparison of Geometric Rectification Methods Based on Forest Satellite Remote Sensing Image
}

\author{
Weiguo LI ${ }^{1,2}$, Wenbing XU² \\ ${ }^{1}$ Shijiazhuang University of Economics, NO.136 Huai an East Road, Shijiazhuang City, Hebei Province, \\ China \\ ${ }^{2}$ Zhejiang Provincial Key Laboratory of Carbon Cycling in Forest Ecosystems and Carbon Sequestration, \\ Zhejiang A \& F University, Lin'an, Hangzhou, Zhejiang, China
}

\begin{abstract}
ABSTRACT: Forest remote sensing image computer processing is a technology used for improving identification ability of remote sensing image in forestry, conducting forest classification and carbon sink with the aid of electronic computers. Quickbird satellite remote sensing image is a commercial satellite remote sensing image with the highest resolution (up to 0.61 meters) currently in the world. The geometric rectification of remote sensing image is the premise to realize image application. This paper expounds the principles and methods of geometric rectification, contrastively analyzes commonly-used geometric model by high-resolution satellites, i.e. Helmert transformation, affine transformation, linear transformation and quadratic polynomial transformation. Experiments show that the quadratic polynomial transformation is feasible with relatively high rectification accuracy for flat terrains.
\end{abstract}

KEYWORD: Remote sensing image; Geometric Rectification; accuracy analysis

\section{INTRODUCTION}

Forest remote sensing image computer processing is a technology used for improving identification ability of remote sensing image in forestry, conducting forest classification and carbon sink with the aid of electronic computers. This technology can not only improve the quality of the remote sensing images and visual interpretation accuracy, but also obtain the number and surface data of forest types and volume. High-resolution satellite remote sensing is the basic space data to constitute a digital Earth and can serve as the carrier and framework for other non-spatial data so as to realize the spatial orientation of a digital Earth[1]. So far in the world, the QuickBird satellite, launched by American DigitalGlobal Company in Oct 18, 2001, is of the highest spatial resolution (up to 0.61 meters) among numerous commercial remote sensing satellites. The QuickBird remote sensing technology is of wide application and the application depth is strengthened continuously.

The geometric rectification of remote sensing image is the premise to realize image application, aiming at rectifying the live map for the spatial distribution of targets on the remote sensing image. It is actually to adopt a mathematical model and choose a certain number of featured points of known theoretical coordinate values to obtain the coordinate values on the base maps, then by using method of undetermined coefficients to calculate the parameters with the transformation from base map coordinates to theoretical coordinates, to further transform the coordinate values of elements to those of required coordinate systems[2]. There are a lot of models for geometric rectification of remote sensing image, for the new type with high resolution as like QuickBird, one of the research focus will be the different rectification model and rectification accuracy.

Polynomial transformation[3] [6] is adopted for topographic map update or remote sensing image vectorization, precision analysis of polynomial transformation is described in the Chen's articles[2]. Rectification models are provided by several GIS softwares such as ERDAS, CASS and MAPINFO, which can be chosen by people. Surely, a geometric rectification model should be selected according to the product types of remote sensing image, the project scale and application range, the present conditions and precision etc.

In this paper, the precision of four rectification models are selected for assessment on the basis of QuickBird orthorectified product by taking a city's planning project as the background and using Southern CASS software. 


\section{GEOMETRIC RECTIFICATION MODEL}

\subsection{Helmert Transformation}

Helmert transformation is also named 7-Parameter transformation, which includes three translation parameters $T_{X}, T_{Y}, T_{Z}$, three rotation parameters $\omega_{X}$, $\omega_{Y}, \omega_{Z}$ and one scale parameter. Suppose there are two space rectangular coordinate systems based on different benchmarks $O_{A}-X_{A} Y_{A} Z_{A}$ and $O_{B}-X_{B} Y_{B} Z_{B}$, then the function model of Helmert transformation is:

$$
\begin{aligned}
& \left(\begin{array}{c}
X_{B} \\
Y_{B} \\
Z_{B}
\end{array}\right)=\left(\begin{array}{c}
T_{X_{A, B}} \\
T_{Y_{A, B}} \\
T_{Z_{A, B}}
\end{array}\right)+\left(1+m_{A, B}\right) \\
& \times\left(\begin{array}{ccc}
1 & \omega_{Z_{A, B}} & -\omega_{Y_{A, B}} \\
-\omega_{Z_{A, B}} & 1 & \omega_{X_{A, B}} \\
\omega_{Y_{A, B}} & -\omega_{X_{A, B}} & 1
\end{array}\right)\left(\begin{array}{c}
X_{A} \\
Y_{A} \\
Z_{A}
\end{array}\right)
\end{aligned}
$$

This model is commonly used in conducting datum transformation worldwide or in a large range, however, since the rotation center is located in the origin of the original coordinate frame of reference, so there are high correlation between rotation parameters and translation parameters.

\subsection{Affine Transformation}

Affine transformation is one type of space rectangular coordinate transformation, which is linear transformation from one 2D coordinate to another. In higher geometry, affine transformation refers to the point transformation with unchanged single ratio of associativity and collinearity from plane to itself. In fact, affine transformation is one of the most commonly-used low order geometric transformation, belonging to the rigid transformation and of good mathematical properties. Affine transformation is a relatively simple linear transformation, also the most commonly-used method for geometric rectification. Its expression is:

$$
\left(\begin{array}{c}
X_{B} \\
Y_{B}
\end{array}\right)=\left(\begin{array}{c}
T_{X_{A, B}} \\
T_{Y_{A, B}}
\end{array}\right)+m\left(\begin{array}{cc}
\cos \omega & \sin \omega \\
-\sin \omega & \cos \omega
\end{array}\right)\left(\begin{array}{c}
X_{A} \\
Y_{A}
\end{array}\right)
$$

The features of affine transformation are: after transformation, a straight line is still a straight line, parallel lines are still parallel lines, the length varies with the direction.

Its theoretical basis is under the condition of relatively small filed angle, photography light beam can be regarded as equivalent parallel projection[7], which is suitable for mapping of middle and small scale topographic map.

\subsection{Linear Transformation}

In the course of photogrammetry, internal and external orientation elements must be known so as to get the object space coordinates of image points. Linear transformation doesn't need the value of inner orientation elements and the initial value of exterior orientation elements, but directly establish the algorithm of direct linear relationship between coordinates of image point coordinate instrument and the object space coordinates of corresponding object points. Suppose the geometric distortion of remote sensing image in a small area is linear transformation, when the coordinate of image point is $(x, y)$ and the coordinate of the ground point is $(X, Y)$, its mathematic expression is

$$
\left\{\begin{array}{l}
X=a x+b y+c \\
Y=d x+e y+f
\end{array}\right.
$$

In the above expression: $a, b, c, d, e, f$ represent model parameters.

\subsection{Quadratic transformation and higher-order transformation}

Quadratic transformation and higher-order transformation are usually known as polynomial transformation, which are commonly used in image rectification. The principle of this model is clear and its calculation is simple. This model is of good rectification precision, specific to flat terrains. The basic idea of the algorithm is to avoid the space geometric process of imaging and conduct mathematical simulation for image distortion itself directly. It is assumed that the overall distortion of remote sensing can be regarded as the combined result of translation, scaling, rotation, affine, partial torsion, bending as well as higher-order basic deformation. Thus an appropriate polynomial formula can be used to express the correction of the coordinate relationship between the corresponding points of images. The mathematic model is:

$$
\left\{\begin{array}{c}
X=a_{0}+a_{1} x+a_{2} y+a_{3} x^{2}+a_{4} x y+a_{5} y^{2}+A \\
Y=b_{0}+b_{1} x+b_{2} y+b_{3} x^{2}+b_{4} x y+b_{5} y^{2}+B
\end{array}\right.
$$

In the expression, $A, B$ represents the sum of quadratic and higher order terms. The above expression is a high order transformation equation, any transformation conforming to the above equation is called higher-order transformation. If $A$ and $B$ are not considered in the high order transformation equation, it will be a quadratic transformation. Quadratic transformation is suitable for the original image with nonlinear transformation, at least six pairs of control-point coordinates and the 
corresponding theoretical values are needed to get undetermined coefficients.

\section{ACCURACY ASSESSMENT}

\subsection{INTERNAL ACCORD ACCURACY}

According to the error equation of different mathematical models, public point observation fitting residual $\Delta_{i}$ can be obtained. When coordinates of the known points are regarded as true values, then valuation $\sigma_{0}$ of overall error of unknown parameters' unit weight can be obtained:

$$
\sigma_{0}= \pm \sqrt{\frac{\left[\Delta_{i} \Delta_{i}\right]}{n}}
$$

In the above equation, $n$ represents the number of public points. The overall error of unknown parameters' unit weight reflects the internal accord accuracy of unknown parameters, which can be used for assessing the stability and convergence of mathematic models.

\subsection{External accord accuracy}

By using mathematic models, residual $v_{i}$ and external accord accuracy $m_{i 0}$ of two-dimensional coordinate are obtained from the checkpoints' coordinate $\left(x_{L}, y_{L}\right)$ and its transformed coordinate $\left(x_{t}, y_{t}\right)$ :

$$
\left.\begin{array}{l}
\left.\begin{array}{l}
v_{x}=x_{t}-x_{L} \\
v_{y}=y_{t}-y_{L}
\end{array}\right\} \\
m_{x}= \pm \sqrt{\left[v_{x} v_{x}\right] / n} \\
m_{y}= \pm \sqrt{\left[v_{y} v_{y}\right] / n} \\
m_{0}= \pm \sqrt{m_{x}^{2}+m_{y}^{2}}
\end{array}\right\}
$$

In the above equation, $n$ represents the number of checkpoints, $m_{i 0}$ represents the external accord accuracy, reflecting the accuracy and consistency of mathematic models.

\section{EXPERIMENTAL COMPARISON}

\subsection{Project description}

In this experiment, the orthogonal projection data of QuickBird high resolution commercial remote sensing is adopted as in figure one, Mercator transformation is used for projection transformation, the benchmark is WGS_1984, the scale is 1:2500, the terrain is flat with the area of about 0.4 square kilometers. The operation method is as follows: Five
Leica 1200 GPS receivers are used to lay out GPS control network, RTK collects image control points and checkpoints as in figure 2; then geometric rectification is made and vectorization of remote sensing is conducted by using the Southern CASS software, finally the planning map of this city is submitted with scale 1:1000.

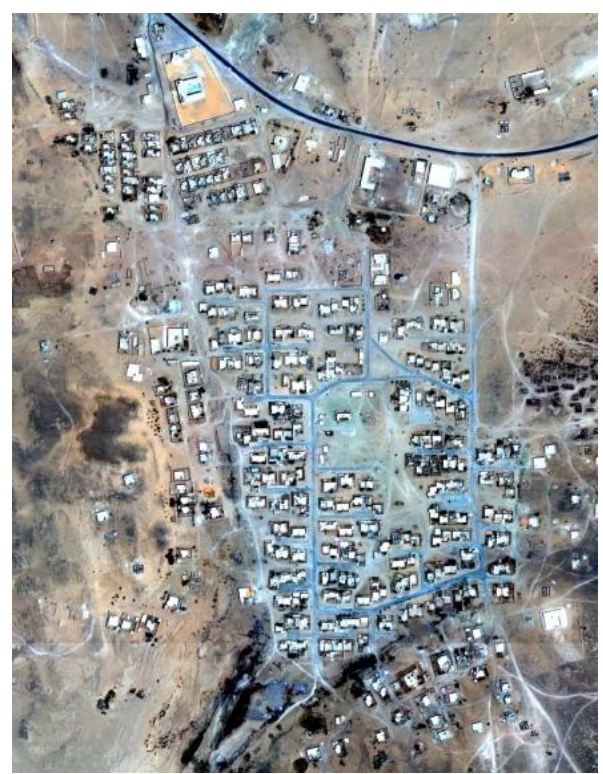

Figure 1 the QuickBird remote sensing image of the city

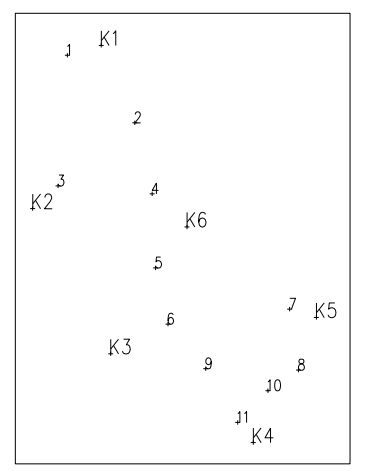

Figure 2 the sketch map of control points and checkpoints

\subsection{Experiment results and accuracy analysis}

In the experimental area, six control points are collected as the common point of the mathematical model and assessment is made about the internal accord accuracy, 12 feature points on the terrain is as checkpoints and the external accord accuracy of mathematical models is evaluated. The four mathematical models adopt the same common points and checkpoints, the result is showed in figure one. The internal and external accord assurance is showed in figure three.

According to the data in the figure and table, the experiment shows the following: firstly, complying with 1:500 1:1000 1:2000 Field Digital Mapping Technology regulations, if mapping is singly used for city planning, the position error should be no more than $\pm 0.50 \mathrm{~m}$ in the topographic map with scale 1:1000[8], Helmert transformation and quadratic 
transformation meet the above regulation and quadratic transformation is of the highest accuracy; secondly, since the features of affine transformation is that its length varies with the orientation, which can lead to a big distortion in one direction, for example, the largest error in $\mathrm{Y}$ direction is up to $1.494 \mathrm{~m}$, consistency and accuracy of external accord accuracy are assured by the homology and collinearity of affine transformation; thirdly, for quadratic transformation and higher-order transformation like cubic transformation, it is said in Chen's articles that there is no obvious difference for the accuracy between cubic and quadratic polynomial, but cubic polynomial obviously takes more time than quadratic polynomial in rectification[2], so we didn't give an analysis on higher-order polynomial in this experiment; fourthly, for the number of parameters, the parameters number of affine transformation and linear transformation is less than Helmert and Quadratic, so less number of control points is need for geometric rectification model, but the accuracy is lower as showed in figure 3; lastly, there is no difference in time cost of rectification for the four transformations.

Table 1 accuracy comparison of different geometric rectification transformation (Unit: m)

\begin{tabular}{|c|c|c|c|c|c|c|c|c|c|c|}
\hline \multirow{3}{*}{$\begin{array}{l}\text { Rectification } \\
\text { model }\end{array}$} & \multicolumn{5}{|c|}{ Internal accord accuracy } & \multicolumn{5}{|c|}{ External accord accuracy } \\
\hline & \multicolumn{2}{|c|}{$\mathrm{X}$} & \multicolumn{2}{|c|}{$\mathrm{Y}$} & \multirow[b]{2}{*}{$\sigma_{0}$} & \multicolumn{2}{|c|}{$\mathrm{X}$} & \multicolumn{2}{|c|}{$\mathrm{Y}$} & \multirow[b]{2}{*}{$m_{0}$} \\
\hline & $\begin{array}{c}\text { Max } \\
\text { Residual }\end{array}$ & $\sigma_{x}$ & $\begin{array}{c}\text { Max } \\
\text { Residual }\end{array}$ & $\sigma_{y}$ & & $\begin{array}{c}\text { Max } \\
\text { Residual }\end{array}$ & $m_{x}$ & $\begin{array}{c}\text { Max } \\
\text { Residual }\end{array}$ & $m_{y}$ & \\
\hline Helmert & 0.502 & 0.372 & -0.467 & 0.282 & 0.467 & -0.409 & 0.287 & 0.651 & 0.360 & 0.460 \\
\hline Affine & 0.552 & 0.347 & -1.494 & 0.663 & 0.748 & -0.463 & 0.249 & 0.651 & 0.315 & 0.401 \\
\hline Linear & 0.427 & 0.280 & -0.951 & 0.490 & 0.565 & -0.740 & 0.368 & 0.928 & 0.339 & 0.500 \\
\hline Quadratic & 0.468 & 0.254 & 0.529 & 0.312 & 0.402 & 0.461 & 0.285 & 0.610 & 0.343 & 0.446 \\
\hline
\end{tabular}

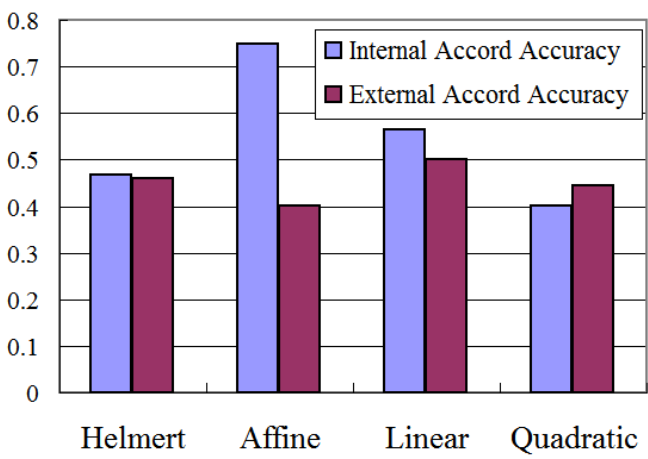

Figure 3 Internal and External Accord Accuracy

There are still a lot of works for further research such as the impact of control points distribution on the accuracy of geometric rectification, the selection of geometric rectification in rugged terrains as well as the error source of field work and laboratory analysis in the process of geometric rectification and so on.

\section{CONCLUSION}

This paper, taking the actual project as the research background and adopting Southern CASS software, compares the four geometric rectification methods, i.e. Helmert transformation, affine transformation, linear transformation and quadratic transformation. It is showed that the accuracy of quadratic transformation mathematic model is better than other transformations in flat terrain; besides, according to the practical experience, the selected control points should be clear and distributed evenly, the control oints of remote sensing image can't be forgotten to select or wrongly selected, the error should be checked whether it meets the accuracy requirement so as to improve the quality and credibility of mapping. Therefore, for the city planning mapping with scale 1:1000, it is an economical, effective and practical method to use QuickBird orthoimage for vectorization.

\section{REFERENCES}

[1] Li Deren \& Li Qingquan. The Earth Space Information Science and a Digital Earth. Advance in Earth Sciences. 1999(12):535 540

[2] Chen Yulin \& Qin Jun. Geometric Rectification of LANDSAT-7 Satellite Image Preprocessing. Railway Investigation and Surveying. 2005(4):16 18.

[3] Bao Jiankuan \& Chen Wenhui. Land Use status mapping Based on QuickBird and GPS RTK. Geomatics \& Spatial Information Technology. 2009(2):25 28.

[4] Cai Hongmei, Li Jinling \& Zhang Jian. Discussion on the technology method of Using QuickBird image to Update 1:2000 Topographic Map.Urban Geotechnical Investigation \& Surveying. 2010(3):85 87.

[5] Zhao Xiuying. Making 1:5000 Topographic Map by using QuickBird Satellite data. Jilin: Jilin University.2005.

[6] Li Shuang etc. Geometric Rectification Accuracy Evaluation in Remote Sensing Mapping. Journal of Capital Normal University. 2008(12):89 92.

[7] Wang Liying. Research on IKONOS Remote Sensing Image Geometric Rectification Methods Based on RFM. Fuxin: Liaoning Project Technology University. 2007.

[8] GB/T 14912-2005, 1:500 1:1000 1:2000 Field Digital Mapping Technology Regulations. 